ISSN: 0212-5374

DOI: http://dx.doi.org/10.14201/et2014321177193

\title{
LA CREDIBILIDAD DE LOS SISTEMAS DE EVALUACIÓN FORMATIVA EN DOCENCIA UNIVERSITARIA: UN ESTUDIO DE CASOS MÚLTIPLE APLICADO
} A LA FORMACIÓN DEL PROFESORADO

\author{
The credibility of formative evaluation systems \\ in university teaching: a multiple case study applied \\ to teacher training
}

\section{La crédibilité des systèmes d'évaluation formative en l'enseignement universitaire: un étude de cas multiples appliqué à la formation du professorat}

Francesc Buscà ${ }^{*}$, M. ${ }^{a}$ del Mar SuÁREZ**, Silvia BuRSET ${ }^{* * * *}$ y Emma BOSCH***** Universidad de Barcelona. Correo-e: *fbusca@ub.edu; **mmsuarez@ub.edu; ****sburset@ub.edu; ******** (emmabosch@ub.edu

Recibido: 24.07.2013; Aceptado: 18.12.2013; Publicado: 31.10.2014 BIBLID [0212-5374 (2014) 32, 2; 177-193]

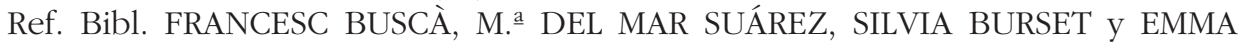
BOSCH. La credibilidad de los sistemas de evaluación formativa en docencia universitaria: un estudio de casos múltiple aplicado a la formación del profesorado. Enseñanza \& Teaching, 32, 2-2014, 177-193.

RESUMEN: El objetivo de este artículo es estudiar diversos sistemas de evaluación formativa implantados en asignaturas de grado y máster adscritas a facultades de formación del profesorado. Para lograr este propósito, se lleva a cabo un plan de trabajo que adopta el formato de un estudio de casos múltiple. En cada uno de estos 
casos, los informadores son, por un lado, el profesorado responsable de la asignatura y, por otro, los estudiantes que han realizado las actividades de enseñanza y aprendizaje y que -incluso- han participado en su evaluación y calificación. Se han utilizado diferentes instrumentos de carácter cualitativo y cuantitativo con la intención de identificar las características principales de los sistemas de evaluación formativa implantados y de constatar los indicadores que permitirán establecer en qué grado los sistemas de evaluación formativa y participativa estudiados son pertinentes y aportan resultados creíbles. Los resultados indican que la credibilidad de estos sistemas de evaluación, además de las calificaciones, también depende de otros indicadores como la calidad del feedback proporcionado por el agente evaluador (docente o estudiante) durante la resolución de las actividades de evaluación, el empoderamiento y la capacidad de estos sistemas para contribuir al desarrollo profesional de los estudiantes. La principal conclusión que se desprende de este estudio se refiere al hecho de que las calificaciones académicas no pueden ser consideradas como el único factor a tener en cuenta para valorar la conveniencia de la evaluación formativa y participativa en docencia universitaria. En este sentido, el principal indicador de la credibilidad de un sistema de evaluación es su capacidad para aportar evidencias del fomento y la adquisición de aprendizajes profundos y competenciales.

Palabras clave: evaluación formativa, evaluación auténtica, evaluación formadora, docencia universitaria, formación del profesorado, EEEs.

SUMMARY: This study aims at analyzing some formative assessment systems applied in some degree and masters connected with teacher training following a multiple case study design. In each case, the informants were, on the one hand, the teacher in charge of the subject and, on the other hand, the students who did the teaching and learning activities and who even participated in the evaluation and assessment of these activities. Several qualitative and quantitative instruments were administered in order to identify the main characteristics of the formative evaluation systems introduced and as certain the indicators that will lead to establish up to what extent the formative and participative evaluation systems studied are appropriate and provide credible results. The results show that the credibility of these evaluation systems, regard less of the students' final marks, depend so not her indicators, such as the quality of the feedback supplied by the person evaluating (either the teacher or the students themselves) in the evaluation activities, the empowerment and the capability of these systems to contribute to the students' professional development. The main conclusion drawn from this study is that academic assessment should not be the only factor taken into account to evaluate the benefits of formative and participative evaluation systems in university teaching. In this regard, the main indicator of the credibility of any evaluation system is its power to provide evidence of the development and acquisition of competences and deep learning.

Key words: formative assessment, authentic evaluation, formative evaluation, teacher training, EHEA. 
FRANCESC BUSCÀ, M. ํㅡㄹ DEL MAR SUÁREZ, SILVIA BURSET Y EMMA BOSCH

RÉSUMÉ: L'objectif de cet article est étudié des systèmes d'évaluation formative implémentés en matières de grade et master liées à la formation du professorat. Pour réussir à ce but, on a conduit un plan de travail qui adopte le format d'une étude de cas multiple. En chacun de ces cas, les informateurs sont, d'un côté, le professorat responsable de la matière et, de l'autre, les étudiants qui ont fait les activités d'enseignement et apprentissage et qui ont même participé en l'évaluation et la qualification. On a distribué des instruments différents de nature qualitative et quantitative dans l'intention d'identifier les caractéristiques principales des systèmes d'évaluation formative implémentés et de constater les indicateurs qui vont permettre établir dans quel dégrée les systèmes d'évaluation formative et participative étudiés sont pertinentes et nous fournissent des résultats crédibles. Les résultats indiquent que la crédibilité de ces systèmes d'évaluation, indépendamment des qualifications des étudiants, dépend aussi d'autres indicateurs comme la qualité du feed-back procuré par l'agent évaluateur (soit l'enseignant, soit l'étudiant) pendant la résolution des activités d'évaluation, la responsabilisation et la capacité de ces systèmes pour contribuer au développement professionnel des étudiants. La principale conclusion de cette étude montre que les qualifications académiques ne peuvent pas être considérées le seul facteur que l'on devrait tenir en compte pour évaluer la convenance de l'évaluation formative et participative en l'enseignement universitaire. Dans ce sens, le principal indicateur de la crédibilité d'un système d'évaluation est sa capacité pour apporter des évidences du développement et de l'acquisition des apprentissages profonds et compétenciaux.

Mots clés: contrôle des connaissances, évaluation authentique, évaluation formative, enseignement universitaire, formation du professorat, EEES.

\section{La evaluación en el marco del Espacio Europeo de Educación Superior}

El proceso de Convergencia Europea supone un cambio en la manera de pensar y concebir la docencia universitaria. En cuanto a la evaluación de los aprendizajes, el Espacio Europeo de Educación Superior (EEES) implica una serie de cambios epistemológicos y metodológicos. En concreto, supone: a) pasar de un paradigma centrado en la figura del docente y de los procesos de enseñanza a otro centrado en el aprendizaje del alumnado, b) desarrollar técnicas e instrumentos de evaluación que vayan más allá de la calificación de los contenidos y que, por tanto, tengan en cuenta los procesos de adquisición de aprendizajes y de competencias, c) desarrollar sistemas de evaluación continuada, formativa y auténtica dirigidos a la mejora de la calidad de los procesos de enseñanza y aprendizaje.

El sistema universitario español no es ajeno a esta transformación. En este sentido, desde hace unos años, ya es posible vislumbrar casos en los que, tanto la política académica ${ }^{1}$ como los proyectos de investigación e innovación que

1. Por ejemplo, las Normas reguladoras de la Evaluación y Calificación de los Aprendizajes de la Universidad de Barcelona recomiendan la adopción de una evaluación continuada, con un enfoque eminentemente formativo y normativo. 
se están llevando a cabo en el marco de la docencia universitaria, contemplan los procesos de evaluación formativa como centro de interés². Todas estas aportaciones confirman que los sistemas alternativos de evaluación están adquiriendo mayor trascendencia en la docencia universitaria (Bain, 2006). Asimismo, también constatan que, en el ámbito de la formación del profesorado, las finalidades formativas derivadas de los planes de estudio centrados en el desarrollo de competencias profesionales disciplinarias y transversales exigen un modelo de evaluación centrado en el aprendizaje de los estudiantes. Esto supone que hay que ir más allá de la calificación de los aprendizajes, y que los docentes universitarios trabajen con metodologías que favorezcan aprendizajes a largo plazo, complejos y profundos (Dochy, Segers y Dierick, 2002).

La necesidad de mejorar las prácticas de evaluación, en combinación con los antecedentes teóricos y prácticos que han aportado autores foráneos en el marco de la docencia universitaria (Biggs, 2005; Boud, 1995; Boud, 1999; Boud y Falchikov, 2007; Falchikov, 2005; Gibbs y Simpson, 2009; OECD/CERI, 2008), ha sido uno de los principales detonantes para disponer de estudios y aportaciones de referencia adecuados al sistema universitario español (Bonsón y Benito, 2005; Bretones, 2008; Fraile, Castejón y Romero, 2013; López, 2004, 2008, 2009; López, Martínez y Julián, 2007; Mateo, 2005; Palacios y López, 2011; Ruiz-Gallardo, Ruiz y Ureña, 2013). Estos referentes están contribuyendo a establecer y a fortalecer una línea de trabajo coherente dentro del campo de la evaluación formativa y participativa. En definitiva, procesos de enseñanza y aprendizaje orientados a formar profesionales reflexivos y críticos, con la capacidad de resolver y transformar las situaciones problemáticas diversas y complejas que deberán afrontar al llevar a cabo su tarea profesional en contextos educativos formales y no formales.

\section{2. ¿QUÉ SE ENTIENDE POR EVALUACIÓN FORMATIVA?}

Tal y como señalan Jorba y Sanmartí (1997), la evaluación formativa es un término que Scriven introdujo en la segunda mitad del siglo pasado, para referirse a los procedimientos que emplea el profesorado con la intención de adecuar su proceso didáctico a los progresos y las necesidades de los estudiantes. Se trata de un enfoque de la evaluación que concibe el aprendizaje como un proceso a largo plazo en el que el estudiante reestructura su conocimiento gracias a las actividades de enseñanza y evaluación que plantean los docentes. La evaluación formativa se refiere, por tanto, a una modalidad de evaluación que tiene como principal función regular el proceso de enseñanza y aprendizaje, y que prioriza la detección de los puntos débiles y mejorar el aprendizaje antes que establecer sus resultados.

2. Por ejemplo, Programas de Innovación y Mejora Docente o Programas de Investigación en Docencia Universitaria. 
La importancia y relevancia de la evaluación formativa en el aprendizaje de los estudiantes es un hecho bastante reconocido. Sin embargo, todas las cualidades de este tipo de evaluación a las que se ha hecho alusión hasta ahora no han sido bien entendidas en el marco de la educación superior (Yorke, 2003), a pesar de que, en un modelo de evaluación centrado en la adquisición de aprendizajes y el desarrollo de competencias profesionales, la evaluación final de los contenidos ya no es tan determinante. Desde este punto de vista, la evaluación va más allá de la calificación o medición del rendimiento. Se convierte en una actividad sistemática de recogida de información con la intención de orientar la mejora de los procesos de enseñanza y aprendizaje en la Universidad (Zabalza, 2002).

Los estudiantes no pueden estar al margen de esta actividad. Si esto fuera así, no se podría atender con suficientes garantías las finalidades y los principios del EEES. Por tanto, la universidad debe asumir que la participación de los estudiantes en los procesos de evaluación es indispensable. Por otra parte, Yorke (2003) señala que la evaluación formativa también debe centrarse en las actividades que realiza el estudiante y que el docente valora proporcionando feedback. Si bien es cierto que la responsabilidad de esta modalidad de evaluación formativa recae en el profesorado, cabe destacar que también puede implicar la participación del alumnado, en el caso de optar por sistemas como la coevaluación o la autoevaluación. Por este motivo, se puede considerar que para iniciar procesos de evaluación formativa y participativa en el marco del EEes y, por extensión, en el marco de una sociedad democrática que pretende formar ciudadanos y profesionales críticos y proactivos, es necesario crear estructuras, procesos democráticos y situaciones de aprendizaje en las que se debe dar cabida a estas prácticas alternativas de evaluación.

\section{LA CREDIBILIDAD DE LOS SISTEMAS DE EVALUACIÓN FORMATIVA EN LA UNIVERSIDAD}

Este artículo tiene como finalidad principal analizar la incidencia de los sistemas de evaluación formativa implementados en diversas asignaturas de grado y máster universitario y, por extensión, constatar la credibilidad de los sistemas de evaluación formativa y participativa en los procesos de enseñanza y aprendizaje llevados a cabo en el marco de la formación del profesorado. Este propósito está justificado por el hecho de que, actualmente, las administraciones estatales y autonómicas centran su interés en constatar la pertinencia y la eficacia de los procesos de enseñanza y aprendizaje llevados a cabo en el seno del sistema universitario.

Dada la dificultad de obtener datos relevantes en un período relativamente corto, parece ser que los únicos indicadores al alcance de las universidades son los siguientes: las calificaciones obtenidas por los estudiantes, la tasa de rendimiento y el número de alumnos que concluyen los estudios. Si bien es cierto que hay que rendir cuentas ante la sociedad, ya sea para justificar tanto la inversión de recursos como las políticas adoptadas en la formación universitaria, hay que insistir también 
en la necesidad de ir más allá de los sistemas de evaluación que sólo se centran en los resultados como único indicador de calidad (Dary, 2010). Solo así se puede garantizar la obtención de una imagen más real de la eficacia y la pertinencia de los servicios y de los procesos formativos que se proporcionan y generan en el seno de las facultades de cualquier universidad.

Glasner (2010) constata que la evaluación en la universidad, independientemente del área de conocimiento, suele utilizar los mismos métodos para evaluar y calificar los estudiantes: pruebas escritas, trabajos en grupo o trabajos prácticos. Ante este hecho, la misma autora propone la utilización de otros métodos o técnicas que complementen el uso de estas prácticas más tradicionales, sobre todo en aquellas asignaturas más nuevas y que, por tanto, todavía no dependen del peso de las convenciones ni los enfoques eminentemente disciplinarios. Para Jorba y Sanmartí (1997) concebir la evaluación como el eje vertebrador de los procesos de enseñanza y aprendizaje es el factor clave para garantizar la calidad de los sistemas de evaluación.

Por contra, el principal argumento que se suele utilizar para poner en duda la pertinencia de este cambio de enfoque de los procesos de evaluación hace referencia a su supuesta dificultad para garantizar su credibilidad. La evaluación formativa es concebida como una modalidad de evaluación cercana a los modelos pedagógicos centrados en las necesidades, los intereses y los derechos del alumnado. Probablemente, este hecho sea el principal argumento esgrimido para desacreditar esta modalidad de evaluación ante los sistemas de evaluación más tradicionales: se considera poco adecuado para constatar el grado de adquisición de competencias y de contenidos académicos. En otras palabras, no sería una modalidad de evaluación pertinente para aquellos modelos curriculares centrados en la figura del docente y en los deberes de los estudiantes. Por todo ello, es importante buscar estrategias para evitar que la asignación de recursos para las universidades dependa cada vez más de los resultados académicos, pues estos promueven la generación de sistemas de evaluación masiva, excesivamente dependientes de la aplicación de una evaluación fundamentada en los criterios de evaluación establecidos de modo acorde.

Las nuevas tendencias en docencia universitaria apuestan por un modelo de evaluación estrechamente vinculado al concepto de evaluación formativa. Se trata de un enfoque que acoge diversas finalidades derivadas de manifestaciones relacionadas con las nociones de:

- Evaluación auténtica, dado que una de las finalidades de la evaluación formativa es aportar evidencias sobre la competencia de los estudiantes para resolver actividades similares a las que deberá realizar en un futuro próximo (Mateo, 2005).

- Evaluación para el aprendizaje y evaluación formadora: de ambos conceptos se recoge el propósito de centrar la evaluación en el aprendizaje del estudiante y autorregular su propio proceso de aprendizaje (López, 2009). 
FRANCESC BUSCÀ, M. a DEL MAR SUÁREZ, SILVIA BURSET Y EMMA BOSCH

\section{PRESENTACIÓN DE LA INVESTIGACIÓN}

De lo visto hasta el momento, se deduce que el concepto de evaluación formativa es mucho más complejo de lo que parece. Si bien podría definirse como aquella modalidad de evaluación que contribuye al aprendizaje del estudiante, conviene no olvidar que tras esta acepción hay toda una serie de condicionantes ontológicos y epistemológicos que determinan su puesta en práctica y su valor pedagógico.

Por este motivo, el objeto de este artículo se ha centrado en estudiar la credibilidad de los sistemas de evaluación formativa llevados a cabo en el marco de la docencia universitaria. Teniendo en cuenta esta finalidad, los objetivos específicos del estudio han sido los siguientes:

a) Explicar los resultados académicos obtenidos mediante los sistemas de evaluación formativa aplicados en algunas de las asignaturas de los grados y másteres que se ofertan en facultades de formación del profesorado.

b) Identificar y describir los factores que determinan la pertinencia y consistencia de los sistemas de evaluación formativa analizados en este estudio.

La investigación adoptó el formato de un estudio de caso múltiple (Stake, 1998). Cada caso se correspondió con una asignatura de grado o máster. Se definieron 6 casos. La Tabla 1 sintetiza los rasgos esenciales de cada uno de ellos.

Durante la implementación de los sistemas de evaluación formativa se aplicaron diferentes instrumentos para la recogida de datos. Todos los casos combinaron estrategias cualitativas (notas de campo elaboradas por el profesorado responsable de la asignatura y/o entrevistas semiestructuradas dirigidas a los estudiantes) con el control de los resultados académicos obtenidos por los estudiantes.

El procedimiento empleado para el tratamiento de los datos fue el siguiente: en primer lugar, se llevó a cabo un análisis descriptivo e interpretativo del contenido de las notas de campo y las entrevistas realizadas durante el período de ejecución del estudio. Este análisis ha sido asistido por el software de análisis de datos cualitativos NVivo 10, y se centró en destacar los elementos más significativos con respecto al impacto de los sistemas de evaluación en los resultados de aprendizaje de los estudiantes. En segundo lugar, este análisis de contenido fue complementado con el análisis estadístico de las calificaciones obtenidas por los estudiantes. Aparte del cálculo de los descriptores básicos, esto es, el promedio e índices de dispersión como la Desviación Estándar (DE) y el Coeficiente de Variación (CV), se extrajeron los estadísticos inferenciales básicos (Chi cuadrado-Chi2 e índice de correlación de Pearson), con la intención de captar el grado de relación existente entre las calificaciones obtenidas y algunos elementos más característicos de los sistemas de evaluación formativa: evaluación continuada y actividades de evaluación. 
TABLA 1

Casos del estudio

\begin{tabular}{|c|c|c|c|c|}
\hline CASO & PARTICIPANTES & TITULACIÓN & ASIGNATURA & CENTRO $^{3}$ \\
\hline FBD & $\begin{array}{l}1 \text { docente } \\
71 \text { estudiantes }\end{array}$ & $\begin{array}{l}\text { Educación Primaria } \\
\text { (grado) }\end{array}$ & $\begin{array}{l}\text { Didáctica de la } \\
\text { educación física }\end{array}$ & $\begin{array}{l}\text { Facultad de Formación } \\
\text { del Profesorado } \\
\text { (Britania) }\end{array}$ \\
\hline MCB & $\begin{array}{l}2 \text { docentes } \\
26 \text { estudiantes }\end{array}$ & $\begin{array}{l}\text { Educación Primaria } \\
\text { (grado) }\end{array}$ & Prácticas & $\begin{array}{l}\text { Facultad de Formación } \\
\text { del Profesorado } \\
\text { (Britania) }\end{array}$ \\
\hline MMH & $\begin{array}{l}2 \text { docentes } \\
76 \text { estudiantes }\end{array}$ & $\begin{array}{l}\text { Ciencias de la } \\
\text { Actividad Física y el } \\
\text { Deporte (grado) } \\
\end{array}$ & $\begin{array}{l}\text { Enseñanza de la } \\
\text { actividad física y } \\
\text { el deporte }\end{array}$ & $\begin{array}{l}\text { Facultad de Educación } \\
\text { (Venetia) }\end{array}$ \\
\hline SBB & $\begin{array}{l}1 \text { docente } \\
27 \text { estudiantes }\end{array}$ & $\begin{array}{l}\text { Educación } \\
\text { Interdisciplinaria de } \\
\text { las Artes (máster) }\end{array}$ & $\begin{array}{l}\text { Comunicación } \\
\text { artística y } \\
\text { aprendizaje } \\
\end{array}$ & $\begin{array}{l}\text { Facultad de Formación } \\
\text { del Profesorado } \\
\text { (Britania) }\end{array}$ \\
\hline EBA & $\begin{array}{l}1 \text { docente } \\
12 \text { estudiantes }\end{array}$ & $\begin{array}{l}\text { Educación } \\
\text { Interdisciplinaria de } \\
\text { las Artes (máster) }\end{array}$ & $\begin{array}{l}\text { Álbum ilustrado: } \\
\text { Significación de la } \\
\text { narrativa gráfica }\end{array}$ & $\begin{array}{l}\text { Facultad de Formación } \\
\text { del Profesorado } \\
\text { (Britania) }\end{array}$ \\
\hline MSV & $\begin{array}{l}1 \text { docente } \\
32 \text { estudiantes }\end{array}$ & $\begin{array}{l}\text { Comunicación } \\
\text { Audiovisual (grado) }\end{array}$ & $\begin{array}{l}\text { Expresión oral y } \\
\text { escrita en inglés }\end{array}$ & $\begin{array}{l}\text { Facultad de } \\
\text { Biblioteconomía } \\
\text { y Documentación } \\
\text { (Britania) } \\
\end{array}$ \\
\hline
\end{tabular}

La presentación de resultados se ha organizado con la intención de dar respuesta a los siguientes interrogantes: ¿Los sistemas de evaluación formativa analizados han contribuido a la adquisición de aprendizajes relevantes por parte de los estudiantes? ¿Se puede afirmar que las evidencias de aprendizaje destacadas por los docentes son creíbles y pertinentes?

\section{RESUltados Y Discusión}

Los sistemas tradicionales de evaluación suelen basarse en la realización de una prueba escrita (o examen) como actividad principal de evaluación. En este tipo de pruebas, los indicadores de aprendizaje se centran exclusivamente en las calificaciones numéricas obtenidas por los estudiantes. Sin embargo, los procesos de evaluación formativa, al requerir actividades más complejas, permiten considerar criterios de evaluación que aportan evidencias de aprendizaje más allá de los resultados académicos.

3. Para preservar el anonimato, el verdadero nombre de las universidades participantes en el estudio ha sido substituido por el nombre de provincias romanas. 
Las principales evidencias de aprendizaje deducidas a partir del análisis de contenido de las notas de campo y las entrevistas semiestructuradas han sido el empoderamiento de los estudiantes; la veracidad de las calificaciones académicas obtenidas a través de los sistemas y las actividades de evaluación formativa; la incidencia del feedback proporcionado por el profesorado; y su contribución al desarrollo de las competencias profesionales de los estudiantes.

\subsection{Una modalidad de evaluación que empodera a los estudiantes}

Tal y como recuerdan Bordas y Cabrera (2001), el empoderamiento se refiere a la incidencia del proceso de evaluación en el desarrollo profesional de los estudiantes que han participado en estos sistemas de evaluación participativa.

En el caso que nos ocupa, los resultados seleccionados en la Tabla 2 pusieron de manifiesto que, a pesar de disponer de la información necesaria para obtener buenas calificaciones, los estudiantes eran conscientes de que, para lograrlo, era necesario esforzarse y seguir las indicaciones del profesorado. Esta percepción fue corroborada por el profesorado, que percibió una mejora progresiva de los procesos de aprendizaje de los estudiantes y, por extensión, de los resultados obtenidos.

TABLA 2

Evidencias del empoderamiento de los sistemas de evaluación formativa estudiados

La manera de evaluar es la que te hace trabajar para que puedas alcanzar los objetivos de la asignatura (Caso FBD).

Los resultados fueron muy satisfactorios, pues los estudiantes fueron capaces de reflexionar sobre sus objetivos y ver que habían podido expresarlos a través de distintas muestras de aprendizaje cuya calidad aumentaba con el tiempo, gracias también al feedback de la profesora (Caso MSv).

Las actividades de evaluación, cuando forman parte del proceso de enseñanza y aprendizaje, es decir, cuando son formativas y formadoras, predisponen al alumnado a aprender y, por extensión, potencian el logro de los resultados de aprendizaje previstos el plan docente de la asignatura (Caso MCB).

La evaluación formativa y participativa es algo más que una modalidad alternativa de evaluación. Implica cambios en la manera de concebir y llevar a cabo las prácticas de evaluación. Pero sobre todo implica cambios de tipo personal a partir del momento en que se opta por implementar y normalizar la participación del alumnado en el proceso de evaluación de su aprendizaje, del propio proceso de enseñanza y aprendizaje o incluso del profesorado. Esta percepción de la evaluación coincide plenamente con las conclusiones obtenidas en los estudios realizados por Bretones (2008) o López (2008), en los que precisamente se destaca que los sistemas de evaluación formativa bien implementados son determinantes para 
dotar a los estudiantes de la conciencia y de la función social y transformadora de su futura actividad profesional.

\subsection{La veracidad de las calificaciones obtenidas a través de los sistemas y actividades de evaluación formativa}

Las calificaciones obtenidas por los estudiantes suelen ser el indicador predominante para discutir la credibilidad de los sistemas de evaluación formativa. Las principales críticas consisten en cuestionar la veracidad de las calificaciones, argumentando que las actividades de evaluación y sus criterios de calificación suelen ser poco rigurosos o demasiado subjetivos (Biggs, 2005; Brown, 2010; López, 2008). Pero ¿es esto cierto?

Un análisis global de las calificaciones obtenidas por los estudiantes en el marco de este estudio permite observar lo siguiente (Figura 1):

- $\quad$ El notable es la calificación que ha obtenido un mayor porcentaje de estudiantes (47\%).

- El porcentaje de aprobados y de excelentes es similar.

- El número de suspensos (7\%) y de no presentados (5\%) es bastante bajo; vistos en conjunto, no llegan al $15 \%$ de los resultados.

- $\quad$ El porcentaje de matrículas de honor es bastante menor, solo un 1\%.

FIGURA 1

Calificaciones obtenidas por los estudiantes que han seguido un proceso de evaluación formativa

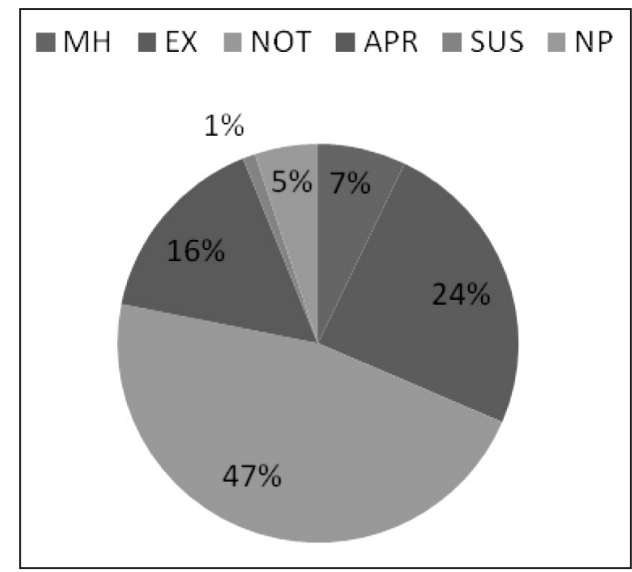

Complementando estos datos con el Coeficiente de Variación (cv), se puede observar que el grado de dispersión de estas calificaciones apenas es apreciable en el caso de los notables y los excelentes y que, con respecto al resto 
de calificaciones, la mayor dispersión de calificaciones se observa en el caso de los suspendidos (Tabla 3). En consecuencia, estos datos permiten insinuar que la mayoría de calificaciones obtenidas en el marco de los sistemas de evaluación de los estudiantes son bastante consistentes. A primera vista, esto puede ser un indicio de que tanto los procesos de evaluación como de calificación han sido rigurosos y de que, por tanto, se han regido por la definición y la aplicación de unos criterios premeditados, y no por el simple capricho del profesorado.

TABLA 3

Dispersión de las calificaciones

\begin{tabular}{|c|c|c|c|}
\hline CALIFICACIONES & PROMEDIO & DE & CV \\
\hline MH & 2,8 & 4,086 & 1 \\
\hline EX & 9,6 & 5,029 & 0 \\
\hline NOT & 18,4 & 11,266 & 0 \\
\hline APRO & 6,2 & 10,158 & 1 \\
\hline SUS & 0,4 & 0,894 & 2 \\
\hline NP & 2 & 3,391 & 1 \\
\hline
\end{tabular}

Para corroborar esta afirmación, hay que tener en cuenta que los datos aportados sobre la distribución de los resultados son bastante equiparables a otros resultados obtenidos en estudios similares (Bretones, 2008; López, 2008, 2009; Fraile et al., 2013). En estos estudios también se observa que el notable es la calificación donde se suelen agrupar la mayoría de los estudiantes, y que el número de suspensos y matrículas de honor es bastante menor.

No obstante, la credibilidad de las calificaciones obtenidas tras la aplicación de los procesos de evaluación formativa también se puede constatar considerando otras evidencias obtenidas del análisis de las calificaciones obtenidas de los casos de este estudio. El primer aspecto a tener en cuenta se refiere a la pertenencia de estos resultados y, por tanto, a si hay motivos objetivos para considerar que las calificaciones obtenidas mediante los procesos de evaluación formativa serían tan válidas como las que - a priori- se obtendrían a través de sistemas de evaluación tradicionales, centrados en el examen. Al realizar esta comparación hipotética (Tabla 4) los resultados obtenidos permiten suponer que estas diferencias no serían apreciables (Chi2: 1,053 para 3 gl, $\mathrm{p}>0,10$ ) y que, por tanto, para establecer en qué grado los sistemas de evaluación formativa proporcionan calificaciones validas y cré́bles, es necesario un análisis más profundo de estos sistemas y considerar otras variables, al margen de los resultados académicos. 
TABLA 4

Distribución de los resultados según la modalidad de evaluación

\begin{tabular}{|l|c|c|c|c|}
\hline $\begin{array}{c}\text { TIPO EVALUACIÓN/ } \\
\text { CALIFICACIÓN }\end{array}$ & MH & EX & NOT & APR \\
\hline Evaluación formativa & 14 & 48 & 92 & 31 \\
\hline Otros sistemas & 46,25 & 46,25 & 46,25 & 46,25 \\
\hline
\end{tabular}

En este sentido, es evidente que los resultados obtenidos han sido bastante satisfactorios. Este hecho se podría justificar porque los sistemas de evaluación formativa permiten: considerar criterios de evaluación vinculados al aprendizaje personal del alumnado y a su capacidad de autorregulación; informar y pactar previamente con los estudiantes los criterios de evaluación y calificación y, gracias a ello, realizar un seguimiento y control continuado del proceso de enseñanza y aprendizaje por parte del profesorado. Estas consideraciones coinciden plenamente con un estudio realizado por la OCDE y centrado en analizar diversas prácticas de evaluación formativa implementadas en el marco internacional (OECD/CERI, 2008). Si bien es cierto que este estudio se focaliza en escuelas de secundaria, los elementos que se destacarían como clave para implementar un sistema pertinente y significativo de evaluación formativa son transferibles al ámbito de la docencia universitaria. Estos elementos serían, entre otros: implicar a los estudiantes en los procesos de aprendizaje y evaluación, emplear métodos de evaluación variados y adaptados a las necesidades de aprendizaje de los estudiantes, administrar feedback adaptado a las situaciones y objetivos de aprendizaje.

Otro factor a considerar tiene que ver con la pertinencia de las actividades de evaluación formativa y, por tanto, con la relación existente entre estas actividades y las calificaciones obtenidas por los estudiantes. En el marco de este estudio, por norma general, ha predominado la realización de dos actividades: el portafolio electrónico y el diseño de propuestas de intervención. Teniendo en cuenta los resultados obtenidos para cada uno de estos instrumentos (Tabla 5), se ha observado una relación directamente proporcional entre estas actividades y las calificaciones obtenidas por los estudiantes ( $r$ : 0,7140 ) y que este grado de asociación no presenta diferencias significativas $(\mathrm{p}>0,05)$. De estos datos se deduce que existirían indicios para afirmar que los resultados obtenidos por ambos tipos de actividad serían pertinentes.

TABLA 5

Calificaciones según el tipo de actividad de evaluación formativa

\begin{tabular}{|l|c|c|c|c|c|c|}
\hline \multicolumn{1}{|c|}{ ACTIVIDAD/CALIFICACIONES } & MH & EX & NOT & APR & SUS & NP \\
\hline Propuestas de intervención & 3 & 33 & 71 & 29 & 0 & 2 \\
\hline eportfolio & 11 & 15 & 21 & 2 & 2 & 8 \\
\hline
\end{tabular}


Este hecho confirmaría también los resultados obtenidos por Palacios y López (2011). Estos autores destacan que los docentes comprometidos por la innovación y la mejora de la docencia suelen utilizar sistemas de evaluación formativa y, por defecto, diseñar actividades e instrumentos de evaluación con mayores probabilidades de obtener resultados de aprendizaje mucho más profundos.

\subsection{El feedback aportado por el profesorado y su incidencia en los resultados y procesos de aprendizaje}

Un tercer criterio a considerar se refiere a la incidencia del feedback proporcionado por el profesorado en los aprendizajes adquiridos por los estudiantes. Tal y como se ha mencionado en el título anterior, el feedback es uno de los elementos más relevantes y significativos de los procesos de evaluación formativa. Su incidencia en los resultados es clave, ya que los estudiantes, para obtener buenas calificaciones, necesitan de la información proporcionada por el profesorado que haga evidentes la relación existente entre la actividad de evaluación y los propósitos de la asignatura, o que les oriente para resolver adecuadamente la actividad de evaluación.

En todos los casos de este estudio, el análisis de los documentos de texto ha permitido constatar que tanto el profesorado como los estudiantes:

- Han sido conscientes de haber emitido o haber recibido feedback durante y después de la realización de las actividades de evaluación.

- Han percibido que este feedback ha sido clave para la obtención de buenos resultados.

Del mismo modo, el análisis de contenido de los informes de cada caso también ha permitido constatar que la información proporcionada por el profesorado, además de incidir en la obtención de resultados satisfactorios, ha contribuido a que los estudiantes sean conscientes de las demandas de las actividades de evaluación, del esfuerzo y de las acciones que se requieren para su resolución (Tabla 6).

Por otra parte, cabe destacar que el alumno suele valorar como positivo el feedback recibido, sobre todo si éste se corresponde claramente con las demandas de la actividad de evaluación y le ayuda a aprobar. Ahora bien, no hay que perder de vista que esta apreciación la suele manifestar una vez ha obtenido una calificación final satisfactoria. En este sentido, no tan solo es evidente que la calidad del feedback es fundamental para obtener buenas calificaciones, sino que además conviene que el profesorado destaque su valor pedagógico. 
TABLA 6

Relación entre el feedback y los resultados de aprendizaje

El hecho de ir haciendo entregas por semanas, el hecho de que las profesoras... pues nos pudierais devolver el trabajo y decirnos en qué puntos flojeábamos, el hecho de poderlo retocar... ha ido muy bien para elaborar un trabajo mucho más completo, mucho más rico y llevar el trabajo al día (Caso MCB).

La manera de evaluar es la que te hace trabajar para que puedas alcanzar los objetivos de la asignatura (Caso FBD).

Los estudiantes muestran un evolución positiva respecto a su autonomía de aprendizaje (Caso MSV).

Todas estas consideraciones con respecto a la calidad y al valor del feedback coinciden con las apreciaciones expuestas por Biggs (2005) con respecto a la importancia de alinear los sistemas de evaluación con los objetivos y las actividades de aprendizaje de la asignatura y, sobre todo, con la capacidad del profesorado para construir a través de su acción docente un clima de confianza y motivación suficiente para que el alumno alcance los resultados de aprendizaje esperados. Del mismo modo, los resultados obtenidos en este apartado se relacionan con las afirmaciones de Gibbs (2010) y Gibbs y Simpson (2009) con respecto a la importancia que el estudiante otorga al feedback que va más allá de la comunicación de los resultados y que, por tanto, está directamente vinculado con la actividad del estudiante.

\subsection{Sistemas de evaluación que contribuyen al desarrollo de competencias profesionales}

En el conjunto de los casos estudiados, se han planteado diversas modalidades de evaluación que han fomentado la participación de los estudiantes. Estas modalidades se han ido desarrollando a lo largo de todo el proceso de evaluación y, en líneas generales, se han caracterizado por proponer a los estudiantes diversas posibilidades de participar en la evaluación, a saber: concretar las actividades de evaluación; negociar con el profesorado los criterios de evaluación y calificación o participar en los procesos de evaluación y calificación. Tal y como se muestra en la Tabla 7, las dinámicas que se generan en los sistemas de evaluación formativos y participativos, además de contribuir a la adquisición de los contenidos de la asignatura y de garantizar la transparencia de los procesos de calificación, también contribuyen al desarrollo de determinadas competencias muy útiles para el posterior desempeño de la profesión. 
FRANCESC BUSCÀ, M. ํㅡㄹ DEL MAR SUÁREZ, SILVIA BURSET Y EMMA BOSCH

TABLA 7

Efectos tangibles de la participación de los estudiantes en los procesos de evaluación

A partir de este tipo de evaluación-metodología, en más de una ocasión algún alumno ha comentado que ha podido extrapolar algunos procesos vividos en la realización de las actividades a otros ámbitos y contextos (Caso SBB).

En la tercera muestra de aprendizaje formal, de la vista 3 del portafolio, los estudiantes fueron incluso más específicos y aportaron evidencias de las acciones de aprendizaje que habían tomado de mayor calidad.

Las reuniones destinadas a concretar y negociar los principales aspectos del sistema de evaluación han estado consideradas como garantía de su transparencia y de la formación como futuros docentes (Caso FBD).

Estas consideraciones irían en la línea de las opiniones expresadas por Dochy et al. (2002) o Gibbs y Simpson (2009). Según estos autores, las evidencias obtenidas tras el análisis de diversos sistemas de evaluación formativa y participativa contribuyen a aceptar la hipótesis de que la credibilidad de estos sistemas no depende tanto de la consistencia las calificaciones académicas como de su capacidad para proporcionar aprendizajes competenciales y profundos.

\section{CONCLUSIONES}

Tras la presentación y discusión de los resultados se puede concluir que:

Las calificaciones académicas no pueden ser consideradas como el único factor a tener en cuenta para valorar la conveniencia de la evaluación formativa y participativa en el marco del EeEs. Si bien es cierto que, atendiendo a la vertiente social de la evaluación, se trata de un aspecto esencial para el sistema universitario, conviene no olvidar que los principios y los fines que se derivan del proceso de convergencia europea también requieren atender a la vertiente pedagógica de la evaluación.

Los resultados alientan a pensar que un proceso de evaluación formativa y participativa, sin estar vinculado a la calificación final, es capaz de promover aprendizajes más profundos. Para que esto sea posible, es necesario proponer e implementar actividades de evaluación participativas, más cercanas a las situaciones profesionales reales y con un alto componente formador tanto por parte del profesorado como del alumnado. Por tanto, es imprescindible replantearse cuestiones referidas a la intención con que se pretende llevar a cabo la evaluación formativa y participativa, los roles y las responsabilidades que se pretende otorgar al alumnado o si la calificación debe ser el eje central del proceso.

Los resultados expuestos en este estudio también han permitido detectar dos vías futuras de estudio. La primera responde a la necesidad de profundizar en el análisis de la carga de trabajo asociada a los sistemas de evaluación formativa. Si 
bien tanto el profesorado como el alumnado participante en este estudio perciben que esta carga es mayor que la que se les supone a los sistemas de evaluación más tradicionales, conviene contrastar esta percepción con una recopilación de datos más rigurosa y sistemática. La segunda línea de estudio se relaciona con la búsqueda de indicadores que corroboren la conveniencia de la evaluación formativa en el marco de la docencia universitaria. La obtención de datos relevantes en cualquiera de estas dos líneas de investigación permitiría obtener más argumentos a favor de esta modalidad de evaluación como la que mejor se ajusta a las directrices del EEES.

\section{REFERENCIAS BIBLIOGRÁFICAS}

Bain, K. (2006). Lo que hacen los mejores profesores universitarios. Valencia: Universidad de Valencia.

Biggs, J. (2005). Calidad del aprendizaje universitario. Madrid: Narcea.

Bonsón, M. y Benito, A. (2005). Evaluación y aprendizaje. En A. Benito y A. Cruz (Eds.). Nuevas claves para la docencia universitaria en el espacio europeo de educación superior (pp. 87-100). Madrid: Narcea.

Bordas, M. I. y Cabrera, F. A. (2001). Estrategias de evaluación de los aprendizajes centradas en el proceso. Revista Española de Pedagogía (Madrid: Instituto Europeo de Iniciativas Educativas) (218), pp. 25-48.

Boud, D. (1995). Enhancing learning through self assessment. London: Routledge Falmer.

Boud, D. (1999). Avoiding the traps: Seeking good practice in the use of self assessment and reflection in professional courses. Social Work Education, 18 (2), 121-132. http://dx.doi.org/10.1080/02615479911220131.

Boud, D. y Falchikov, N. (2007). Rethinking assessment in higher education. Learning for the longterm. Oxon: Routledge.

Bretones, A. (2008). Participación del alumnado de educación superior en su evaluación. Revista de Educación (347), 181-202.

Brown, S. (2010). Estrategias institucionales en evaluación. En S. Brown y A. Glasner (Eds.). Evaluar en la universidad. Problemas y nuevos enfoques (pp. 23-33). Madrid: Narcea.

Dary, E. T. (2010). Evaluación y valoración: Una aproximación sistémica. En S. Brown y A. Glasner (Eds.). Evaluar en la universidad. Problemas y nuevos enfoques (pp. 49-60). Madrid: Narcea.

Dochy, F.; Segers, M. y Dierick, S. (2002). Nuevas vías de aprendizaje y enseñanza y sus consecuencias: Una nueva era de evaluación. Boletín de la Red Estatal de Docencia Universitaria, 2 (2), 12-31.

Falchikov, N. (2005). Improving assessment through student involvement. Practical solutions for aiding learning in higher and further education. Oxon: Routledge.

Fraile, A.; Castejón, F. J. y Romero, R. (2013). La evaluación formativa en docencia universitaria y el rendimiento académico del alumnado. Aula Abierta, 41 (2), 23-34.

Gibbs, G. (2010). Uso estratégico de la evaluación en el aprendizaje. En S. Brown y G. Gibbs (Eds.). Evaluar en la universidad. Problemas y nuevos enfoques (pp. 61-74). Madrid: Narcea.

Gibbs, G. y Simpson, C. (2009). Condicions per a una avaluació continuada que a favor eixi l'aprenentatge. Barcelona: Octaedro-ICE Universitat de Barcelona. 
Glasner, A. (2010). Innovaciones en la evaluación del estudiante: un sistema de amplia perspectiva. En S. Brown y A. Glasner (Eds.). Evaluar en la universidad. Problemas y nuevos enfoques (pp. 35-48). Madrid: Narcea.

Jorba, J. y Sanmartí, N. (1997). La evaluación como instrumento para mejorar el proceso de aprendizaje de las ciencias. En L. D. Carmen (Ed.). La enseñanza y el aprendizaje de las ciencias de la naturaleza en la educación secundaria (pp. 155-199). Barcelona: ICE Universidad de Barcelona, Editorial Horsori.

López, V. (2004). Evaluación, calificación, credencialismo y formación inicial del profesorado: Efectos y patologías generadas en la enseñanza universitaria. Revista Interuniversitaria de Formación del Profesorado, 18 (3), 221-232.

López, V. (2008). Desarrollando sistemas de evaluación formativa y compartida en la docencia universitaria. Análisis de resultados de su puesta en práctica en la formación inicial del profesorado. European Journal of Teacher Education, 31 (3), 293-311. http://dx.doi.org/10.1080/02619760802208452.

López, V. (2009). Evaluación formativa y compartida en educación superior, propuestas, técnicas, instrumentos y experiencias. Madrid: Narcea.

López, V.; Martínez, L. F. y Julián, J. A. (2007). La red de evaluación formativa, docencia universitaria y espacio europeo de educación superior. Presentación del proyecto, grado de desarrollo y primeros resultados. Boletín de la Red Estatal de Docencia Universitaria (2), 1-19.

Mateo, J. (2005). Nou enfocament de l'avaluació dels aprenentatges en el context europeu d'educació superior. En J. Mateo y F. Martínez (Eds.). L'avaluació alternativa dels aprenentatges (1), 7-22. Barcelona: ICE.

OECD/CERI (2008). Assessment for learning formative assessment. Centre for Educational Research and Innovation. Descargado el día 10.07.2013. http://www.oecd.org/site/educeri21st/40600533.pdf.

Palacios, A. \& V. L. (2011). Haz lo que yo digo pero no lo que yo hago: sistemas de evaluación del alumnado en formación inicial del profesorado. Revista de Educación (361), 279-305.

Ruiz-Gallardo, J. R.; Ruiz, E. y Ureña, N. (2013). La evaluación en formación inicial del profesorado: Qué creemos hacer y qué perciben los alumnos. Cultura, Ciencia y Deporte, 8 (22), 17-29.

Stake, R. E. (1998). Investigación con estudio de casos. Madrid: Morata.

Yorke, M. (2003). Formative assessment in higher education: Moves towards theory and the enhancement of pedagogic practice. Higher Education (45), 477-501. http://dx.doi.org/10.1023/A:1023967026413

Zabalza, M. Á. (2002). La enseñanza universitaria. Narcea: Madrid.

Agradecimientos. Este artículo forma parte de la fase de difusión del proyecto de innovación Sistemes d'avaluació formativa i participativa en docència universitària: anàlisi de la seva implantació $i$ impacte en els procesos d'ensenyament $i$ aprenentatge. Este proyecto ha sido subvencionado por la Agència de Gestió d'Ajuts Universitaris i de Recerca (AGAUR) en el marco del programa Millora de la Qualitat Docent (MQD2010 00051). 